\title{
STRUCTURE OF ENDOMORPHISM SEMIGROUP OF ENDOMAPPINGS OF SOME PARTICULAR ROOTED TREES
}

\author{
MOHD. ALTAB HOSSAIN ${ }^{*}$ \\ Department of Mathematics, University of Rajshahi, Rajshahi-6205, Bangladesh
}

\begin{abstract}
For an endomapping of a finite set of points lying on some rooted particular trees, endomorphism and endomorphism semigroup were studied. The main aim of this paper was to obtain the structure of the semigroup of all endomorphisms of the endomappings represented by directed graphs on particular types of rooted trees.
\end{abstract}

Key words: Endomapping, Endomorphism, Endomorphism semigroup, Wreath product, Directed graph

\section{INTRODUCTION}

Very impressive ideas and techniques in semigroups have been studied in the publication Algebraic Theory of Semigroups in 1961 by Clifford and Preston. For being interested in semigroups a number of authors developed this part of mathematics as an important research tool. Some excellent results on inverse semigroups have been presented to reinforce the study of this area by Petrich 1984. A class of additive commutative semigroups of special elements having a unique expression was studied and some characterization properties were found by Majumdar and Hossain (2008). Also, the structure of endomorphism semigroup was obtained by these authors. A feature of the automorphism groups of special semigroup was studied in consequences of earlier work (Hossain 2010). From the interest of getting structures of the endomorphism semigroup of an endomapping of a finite set, a study was made on the semigroup of endomappings directed by graphs of some trees consisting of a chain or chains of equal lengths (Majumdar 2011a).

To determine the structure for endomorphism semigroup of endomappings of some particular rooted trees (almost general case of Majumdar 2011a), considered the semigroup End $f$ for that class of endomappings $f$ such that, for each $x \in X$, there exists a positive integer $r_{x}$ with the property that $f^{r_{x}+1}(x)=f^{r_{x}}(x)$. The technique of structuredetermination consists of :

(i) Representing $f$ by a directed graph $G(f)$ with vertices the points of $X$ and edges $x \rightarrow f(x)$, and

\footnotetext{
*Correspoding author: <al_math_bd@yahoo.com>
} 
(ii) determining the structure of the semigroup $\operatorname{End}(G(f))$ of those transformations $T$ of this directed graph $G(f)$ such that $T(f(x))=f(T(x))$ i.e., $T(x \rightarrow f(x))$ $=(T(x) \rightarrow T(f(x)))$.

Since $T$ maps vertices onto vertices and edges onto corresponding edges, $T$ is called an endomorphism of the digraph of $f$. If $g$ is the endomapping of $X$ induced by $T$, the map $g \rightarrow T$ is an isomorphism of End $f$ into the endomorphism semigroup of $G(f)$. The structure of the transformation semigroup $\operatorname{End}(G(X))$ for a class of endomappings $f$ of some rooted trees is determined through the isomorphism End $f \cong \operatorname{End}(G(X))$.

\section{NECESSARY PRELIMINARIES}

Consider an endomapping $f: X \rightarrow X$ of a finite non-empty set $X$. Under the composition of maps the collection of all endomappings of $X$, denoted by $E(X)$, is a semigroup called the full transformation semigroup on $X$. If the number of elements of $X$ is $n$, one may also write $F_{n}$ for $E(X)$. A map $g: X \rightarrow X$ is called an endomorphism off if $g f=f g$ i.e., if $g$ belongs to the centraliser of $f$ in $E(X)$. The centraliser of $f$, $C(f)=\{g \in E(X) \mid g f=f g\}$, is a transformation semigroup on $X$ called the endomorphism semigroup of $f$ and it is denoted by End $f$. A semigroup $S$ is called a transformation semigroup on a nonempty set $X$, and is written $(S, X)$ if there is a map $S \times X \rightarrow X$ given by $(s, x) \rightarrow x$ such that $\left(s_{1} s_{2}\right)(x)=s_{1}\left(s_{2}(x)\right)$. If $S$ is a monoid, then $1(x)=x$, for each $x \in X$. For transformation semigroups $S_{1}$ and $S_{2}$ on disjoint nonempty sets $X_{1}, X_{2}$, the direct product $S_{1} \times S_{2}$ is a transformation semigroup on $X_{1} \cup X_{2}$ with action given by $\left(s_{1}, s_{2}\right)\left(x_{1}\right)=s_{1}\left(x_{1}\right)$ and $\left(s_{1}, s_{2}\right)\left(x_{2}\right)=s_{2}\left(x_{2}\right)$. For two non-empty sets $X_{1}, X_{2}$, the wreath product $S_{1} \varsigma S_{2}$ is a transformation semigroup on $X_{1} \times X_{2}$ and consists of maps $\theta: X_{1}, X_{2} \rightarrow X_{1}, X_{2}$ given by $\theta\left(x_{1}, x_{2}\right)=\left(s_{1, x_{2}}\left(x_{1}\right), s_{2}\left(x_{2}\right)\right), s_{1, x_{2}}$ being an element of $S_{1}$ determined by $x_{2}$.

To determine the structure of the transformation semigroup $\operatorname{End}(G(X))$ the author needs some results (Majumdar 2011a,b, Meldrum 1995) about the direct product and wreath product of transformation semigroups. The author recalls these in the following way.

The wreath product has a description in terms of direct product which makes the sense that wreath product is associative and is distributive over direct product.

Theorem 1: $\left(S_{1} \varsigma S_{2}, X \times X_{2}\right) \cong\left(\left(\underset{x_{2} \in X_{2}}{\times} S_{1, x_{2}}\right) \times S_{2},\left(\underset{x_{2 \in X_{2}}}{\cup} X_{1, x_{2}}\right) \times X_{2}\right)$ where each $x_{2} \in X_{2}$, $S_{1, x_{2}} \cong S_{1}$ and $\left|X_{1, x_{2}}\right|=\left|X_{1}\right|$

Theorem 2: $\left(\left(S_{1} \varsigma S_{2}\right) \varsigma S_{3},\left(X_{1} \times X_{2}\right) \times X_{3}\right) \cong\left(S_{1} \varsigma\left(S_{2} \varsigma S_{3}\right), X_{1} \times\left(X_{2} \times X_{3}\right)\right)$.

Theorem 3: $\left(S_{1} \varsigma\left(S_{2} \times S_{3}\right), X_{1} \times\left(X_{2} \cup X_{3}\right)\right) \cong\left(\left(S_{1} \varsigma S_{2}\right) \times\left(S_{1} \varsigma S_{3}\right)\right.$,

$$
\left.\left(X_{1} \times X_{2}\right) \cup\left(X_{1} \times X_{3}\right)\right) \text {. }
$$


Remarks: (i) If $S_{2}=\left\{1_{X_{2}}\right\}$, then $\left(S_{1} \times S_{2}, X_{1} \cup X_{2}\right)$ may be identified with $\left(S_{1}, X_{1}\right)$ and $\left(S_{1} \varsigma S_{2}, X_{1} \times X_{2}\right)$ with $\left(\prod_{x_{2} \in X_{2}} S_{1, x_{2}}, \bigcup_{x_{2} \in X_{2}} X_{1, x_{2}}\right)$ where $\left|X_{1, x_{2}}\right|=\left|X_{1}\right|$.

(ii) If $S_{1}=\left\{1_{X_{1}}\right\}$, then both $\left(S_{1} \times S_{2}, X_{1} \cup X_{2}\right)$ and $\left(S_{1} \varsigma S_{2}, X_{1} \times X_{2}\right)$ may be identified with $\left(S_{2}, X_{2}\right)$.

(iii) If $X_{1}=X_{2}=X$, then $\left(S_{1} \varsigma S_{2}, X \times X\right)$ may be identified with $\left(\prod_{x \in X} S_{1, x}\right) \times S_{2}$, $\left.\bigcup_{x \in X} X_{1, x} \cup X\right)$. As semigroups, $S_{1} \varsigma S_{2} \cong\left(\prod_{x \in X} S_{1, x}\right) \times S_{2}$.

\section{STRUCTURE OF THE ENDOMORPHISM SEMIGROUP End $f$}

Author knows about End $f$ through representation of $f$ by $G(f)$, the directed graph of $f$ consisting of a single chain given by Fig. 1:

In this case, End $f$ is the semigroup $E(m)=\left\{\sigma_{0}, \sigma_{1}, \cdots \cdots, \sigma_{m}\right\}$, where $\sigma_{0}$ is the identity element and $\left\{\sigma_{1}, \cdots \cdots, \sigma_{m}\right\}$ is a cyclic semigroup generated by $\sigma_{1}$ with $\sigma_{1}^{m}=\sigma_{m}$ as the zero element.

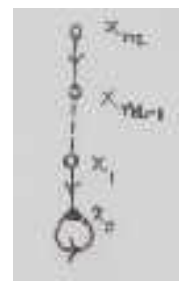

Fig. 1

Let $f$ be given by the directed graph $G(f)$ in Fig. 2:

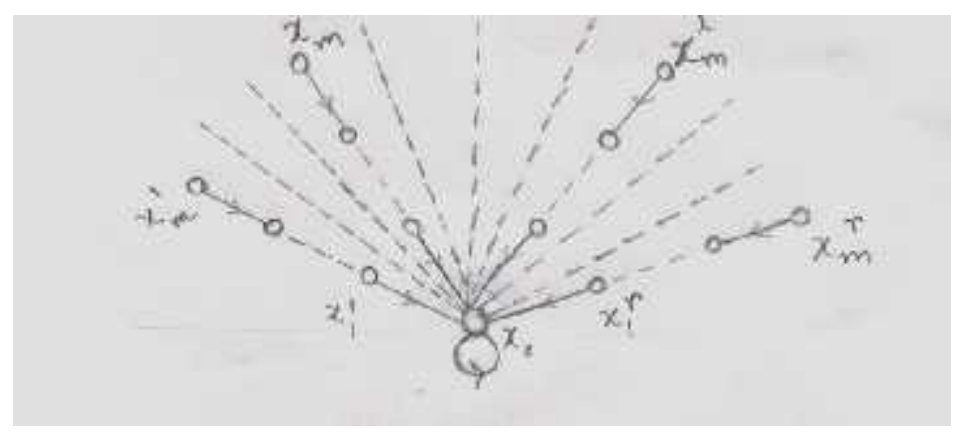

Fig. 2

consisting of $r$ directed subgraphs each being a chain of length $m$ and each with the loops at $x_{0}$. Since each $g \in$ End $f$ must map $x_{0}$ onto itself and since each maximal chain ending at $x_{0}$ has the same length $m$, End $f$ may be identified with the semigroup of all endomorphisms of an endomapping $f^{\prime}$ of $X$ whose directed graph is $C_{m} \times\{1,2, \cdots \cdots, r\}, C_{m}$ being the chains shown in the directed graph. It therefore follows from the theorems of wreath product that $E$ nd $f \cong E(m) \varsigma F_{r}$. Then End $f \cong E(m) \varsigma F_{r} \quad \ldots$ (1). 
Here, $F_{r}$ is the full transformation semigroup on a set with $r$ elements.

It is observed that if $f$ is given by the directed graph $G(f)$ in Fig. 3:

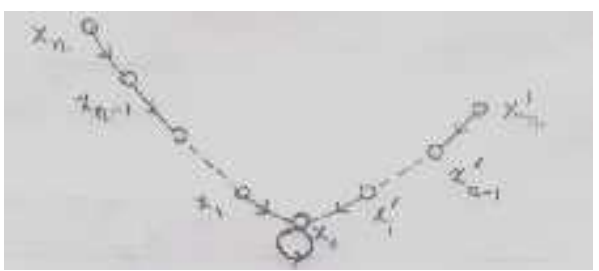

Fig. 3

with $\mathrm{m}>\mathrm{n}$, i.e., the directed graph consists of two chains of unequal lengths, then $\operatorname{End}(X, f)$ will consist of maps:

(a) $T_{1} \rightarrow T_{1}, \quad T_{2} \rightarrow T_{2}$

(a) $T_{1} \rightarrow T_{1}, \quad T_{2} \rightarrow T_{1}$

(a) $T_{1} \rightarrow T_{2}, \quad T_{2} \rightarrow T_{1}$

(a) $T_{1} \rightarrow T_{2}, \quad T_{2} \rightarrow T_{2}, T_{1}$ and $T_{2}$ being subgraphs. Therefore, one may have $\left\{\begin{aligned} \text { End } f= & \left(\operatorname{End} f_{1} \times \text { End } f_{2}\right) \cup\left(\text { End } f_{1} \times \operatorname{Hom}\left(T_{2}, T_{1}\right)\right) \cup \\ & \left(\operatorname{Hom}\left(T_{1}, T_{2}\right) \times \operatorname{Hom}\left(T_{1} \times T_{2}\right)\right) \cup\left(\operatorname{Hom}\left(T_{1}, T_{2}\right), \text { End } f_{2}\right),\end{aligned}\right.$

where, $f_{1}$ and $f_{2}$ are $f$ restricted to $\left\{x_{0}, x_{1}, \cdots, x_{m}\right\}$ and $\left\{x_{0}, x_{1}^{\prime}, \cdots, x_{n}^{\prime}\right\}$, respectively and $\operatorname{Hom}\left(T_{i}, T_{j}\right) \quad(i, j=1,2 ; i \neq j)$ denotes the set of maps the directed graph $T_{i}$ into the directed graph $T_{j}$ of $f_{i}$ and $f_{j}$, respectively.

with

$$
\left\{\begin{array}{l}
\left(\operatorname{End} T_{i}\right) \operatorname{Hom}\left(T_{j}, T_{i}\right) \subseteq \operatorname{Hom}\left(T_{j}, T_{i}\right) \\
\operatorname{Hom}\left(T_{i}, T_{j}\right) \operatorname{Hom}\left(T_{j}, T_{i}\right) \subseteq \operatorname{End} T_{j}
\end{array}\right.
$$

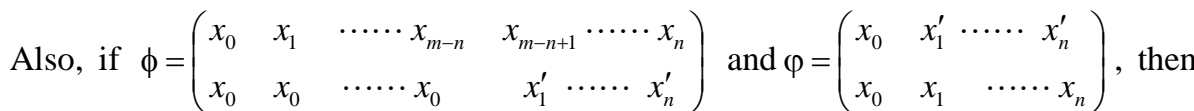
it is easy to see that

$$
\left\{\begin{array}{l}
\operatorname{Hom}\left(T_{1}, T_{2}\right)=\left(\operatorname{End} T_{2}\right) \phi \\
\operatorname{Hom}\left(T_{2}, T_{1}\right)=\left(\operatorname{End} T_{1}\right) \varphi
\end{array}\right.
$$

It follows from the above facts that

$$
\left\{\begin{array}{l}
\left(\operatorname{End} f_{1}\right) \times \operatorname{Hom}\left(T_{2}, T_{1}\right)=\left(\text { End } f_{1}\right)^{2} \varphi, \\
\left(\operatorname{Hom}\left(T_{1}, T_{2}\right)\left(\operatorname{End} f_{1}\right)=\left(\text { End } f_{2}\right) \phi\left(\text { End } f_{1}\right),\right. \\
\left(\operatorname{End} f_{2}\right) \times \operatorname{Hom}\left(T_{1}, T_{2}\right)=\left(\text { End } f_{2}\right)^{2} \varphi, \\
\left(\operatorname{Hom}\left(T_{2}, T_{1}\right)\left(\operatorname{End} f_{2}\right)=\left(\text { End } f_{1}\right) \varphi\left(\text { End } f_{2}\right),\right. \\
\left(\operatorname{Hom}\left(T_{1}, T_{2}\right)\right)\left(\operatorname{Hom}\left(T_{2}, T_{1}\right)\right)=\left(\text { End } f_{2}\right) \phi\left(\text { End } f_{1}\right) \varphi, \\
\left(\operatorname{Hom}\left(T_{2}, T_{1}\right)\right)\left(\operatorname{Hom}\left(T_{1}, T_{2}\right)\right)=\left(\operatorname{End} f_{1}\right) \varphi\left(\operatorname{End} f_{2}\right) \phi .
\end{array}\right.
$$


It, therefore, may be stated that:

Theorem 4: The semigroup-structure of End $f$ of $f$ given by Fig. 3 is completely given by the expressions from (1) to (5).

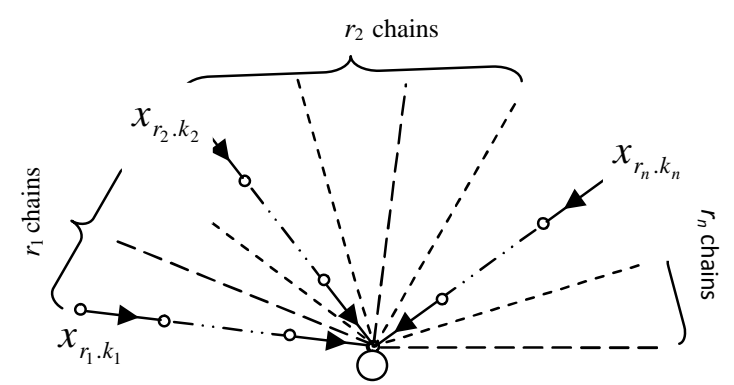

Fig. 4

Theorem 5: Let $f$ be given by the directed graph $G(f)$ as in Fig.4 with $f^{k}(X)$

a singleton, where $k=\max \left\{k_{1}, \cdots, k_{m}\right\}$. Then

(6) End $f=\bigcup\left[\right.$ End $T^{i_{1}} \times \cdots \cdots \times$ End $\left.T^{i_{u}} \times\left(\times \frac{v \neq v^{\prime}}{v \neq v, v^{\prime}<k} \operatorname{Hom}\left(T^{i_{v}}, T^{i_{v^{\prime}}}\right)\right)\right]$,

the union being taken over all permutations $\left(\begin{array}{cccc}1 & 2 & 3 & \cdots \cdots k \\ i_{1} & i_{2} & i_{3} & \cdots \cdots i_{k}\end{array}\right)$.

Here,

(7) $\left\{\begin{array}{l}\operatorname{End} T^{i}=\operatorname{End}^{i, \alpha} \zeta F_{i_{r}} \quad\left(\alpha \in\left\{1, \quad 2, \quad 3, \cdots \cdots, \quad i_{r}\right\}\right), \\ \operatorname{Hom}\left(T^{i_{v}}, T^{i_{v^{\prime}}}\right)=\times_{1 \leq \beta \leq r_{i_{v^{\prime}}}} \operatorname{Hom}\left(T_{\alpha}^{i_{v}}, T_{\beta}^{i_{\gamma^{\prime}}}\right) .\end{array}\right.$

The products of End $T^{i, \alpha}$ with themselves and with $\operatorname{Hom}\left(T_{\alpha}^{i}, T_{\beta}^{j}\right)$ as well as the products of $\operatorname{Hom}\left(T_{\alpha}^{i}, T_{\beta}^{j}\right)$ among themselves are given by (5). Also, the End $T^{i, \alpha}$, s are isomorphic to one another, since $T^{i, \alpha}$, s are chains of the same length.

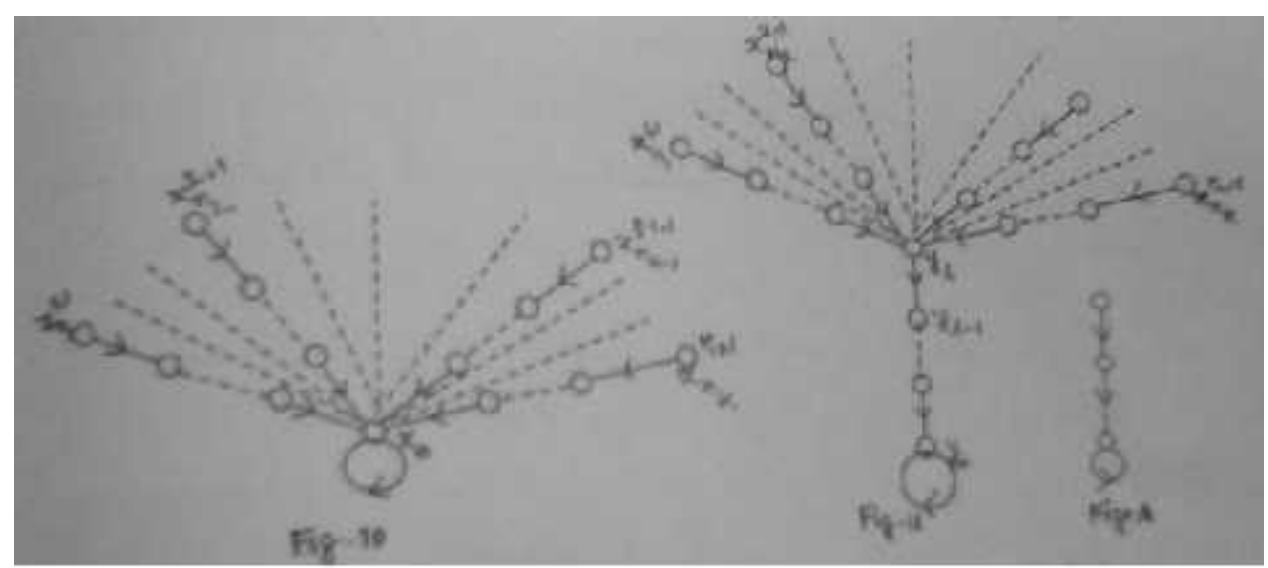


One may consider the situation that the directed graph representing the endomapping consists of a finite number of disjoint directed rooted trees shown as above:

Now let $(X, f)$ be given by the directed graph in Fig. 11. Then a map $g: X \rightarrow X$ is in $\operatorname{End}(X, f)$ if and only if $g$ induces an endomorphism of chain $\mathrm{C}$ [fig.A] and two such endomorphisms combine to yield an element of $\operatorname{End}(X, f)$. It is noted that the endomorphism semigroup of the graph of $(X, f)$ in this case and that of the directed rooted tree with root at $y_{0}$ obtained by removing the loop at $y_{0}$ are isomorphic to each other. Thus, if one denotes $\operatorname{End}(X, f)$ in fig.10 by $E$, then $\operatorname{End}(X, f)$ in Fig.11 is given by $\operatorname{End}(X, f) \cong E \times E(l)($ direct product), $E(l)$ being the endomorphism semigroup of chain $C$.

Let $(X, f)$ be now given by a more general form Fig. 12

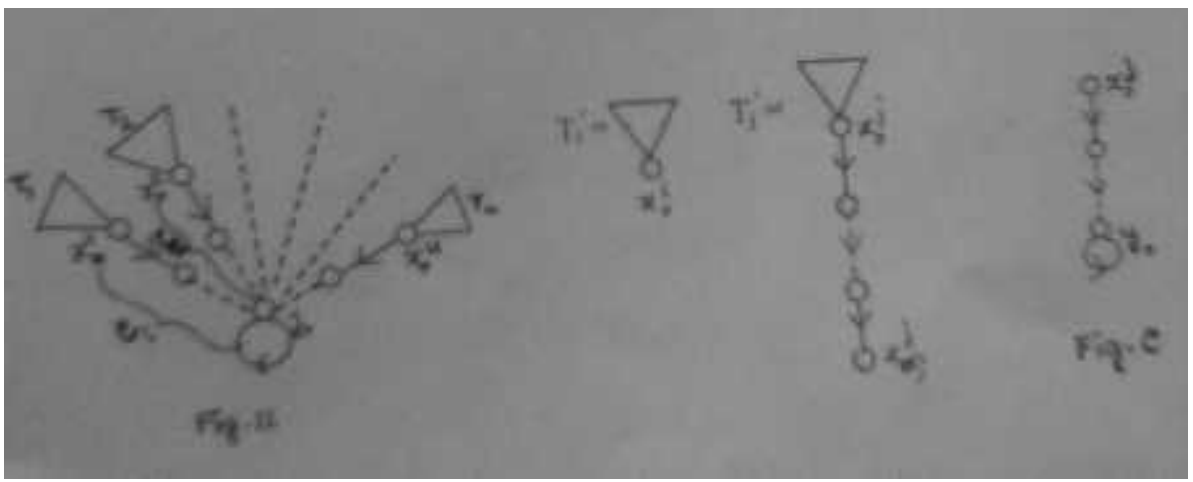

where $T_{1}, \ldots, T_{u}$ are graphs of the form in the earlier case, which are isomorphic to directed trees each with root at $y_{0}$. An endomorphism of $(X, f)$ maps every tree $T_{i}$ into a tree $\mathrm{T}_{\mathrm{j}}(i, j$ not necessarily distinct) in such a way that

(a) $y_{0}$ is mapped onto $y_{0}$,

(b) $x_{i}^{0}$ is mapped onto a point in $\mathrm{C}$, where $\mathrm{C}$ is shown in the Fig. $\mathrm{C}$.

the subtree $T_{i}^{\prime}$ of $T_{i}$ into the subtree $T_{j}^{\prime}$, where $x_{\alpha_{j}}^{j}=f\left(x_{0}^{i}\right)$ is such that the directed edges are mapped onto the corresponding directed edges.

Thus,

(6) $\quad \begin{aligned} & \operatorname{End}(X, f) \\ & =\bigcup\left[\operatorname{End} T^{i_{1}} \times \cdots \cdots \times \operatorname{End} T^{i_{u}} \times\left(\times_{u<v, v^{\prime}<k}^{v \neq v^{\prime}} \operatorname{Hom}\left(T^{i_{v}}, T^{i_{v^{\prime}}}\right)\right)\right],\end{aligned}$

the union being taken over all permutations $\left(\begin{array}{cccc}1 & 2 & 3 & \cdots \cdots k \\ i_{1} & i_{2} & i_{3} & \cdots \cdots \\ i_{k}\end{array}\right)$.

Here, it is easily verified that

(i) if the length $l_{i}$ of $C_{1}$ is greater than or equal to the length $l_{j}$ of $C_{2}\left(C_{1}\right.$ and $C_{2}$ are indicated in the Fig. 12), then $\operatorname{Hom}\left(T_{i}, T_{j}\right)=\left\{\left(\sigma_{j, 2}, \sigma_{j, 1}\right)\left(f_{i j}, \sigma_{i, 1}\right)\right\}$, 
where $f_{i j}=\left(\begin{array}{ccccc}x_{0}^{i} \cdots & x_{l_{1}, 2}^{i} \cdots & x_{l_{i}, s}^{i} & y_{0} \\ x_{0}^{j} & x_{l_{1}, 2}^{j} & \cdots & x_{l_{j}}^{j} & y_{0}\end{array}\right)$,

$\sigma_{i, 1} \in \operatorname{End}\left(T_{i}^{\prime}\right), \quad \sigma_{j, 2} \in \operatorname{End}(C), \quad \sigma_{j, 2} \in \operatorname{End}\left(T_{j}^{\prime}\right)$, (as in the Fig. C).

(ii) if $l_{i} \leq l_{j}$, then $\operatorname{Hom}\left(T_{i}, T_{j}\right)=\left\{\left(\sigma_{j, 2}, \sigma_{j, 1}\right)\left(f_{i j}^{\prime}, \sigma_{i, j}^{\prime}\right)\right\}$

In the above, $f_{i j}^{\prime}: T_{i} \rightarrow T_{j}$ maps every subgraph of $T_{j}$ which is a chain or tree of length $\leq l_{j}$ with root $x_{0}^{i}$ onto the subgraph $C$ (as in the Fig. C) of $T_{j}$ isomorphically.

Also, $\sigma_{i, 1}^{\prime} \in$ End $T_{i}^{\prime \prime}$, where $T_{i}^{\prime \prime}$ is the directed rooted tree obtained from $T_{i}^{\prime}$ by (a) deleting the maximal chain-subtrees in $T_{i}$ with root $x_{0}^{i}$ and length $<l_{j}$ and (b) collapsing the chain-subtrees of $T_{i}$ with root $x_{0}^{i}$ and length $\geq l_{j}$ by identifying all of $x_{0}^{i}, x_{i}^{i}, \cdots, x_{l_{j}}^{i}$ so that they represent the same point while the directed edges $x_{\alpha+1} x_{\alpha}\left(0 \leq \alpha \leq l_{j_{1}}\right)$ vanish.

Multiplication of the elements of $\operatorname{Hom}\left(T_{i}, T_{j}\right)$ with those of $\operatorname{Hom}\left(T_{j}, T_{k}\right)$ is the composition of the maps representing the elements; and multiplication of the elements of End $T_{1} \times \cdots \times$ End $T_{n}$ with the elements of $\operatorname{Hom}\left(T_{i}, T_{j}\right)$ is defined similarly.

\section{CONCLUDING REMARK}

Structures for endomorphism semigroup of endomappings given by directed graphs of some particular rooted trees are almost general case that Majumdar (2011) studied. Such a study for general case, though it is complicated, will be taken up in future.

\section{REFERENCES}

Clifford, A. H. and G. B. Preston. 1961. The algebraic theory of semigroups. Amer. Math. Soc. New York.

Hossain, M. A. 2010. The automorphism group of special semigroups. J. math. Sci. 25: 9-12.

Majumdar, S. and M. A. Hossain. 2008. The endomorphism semigroup of a special semigroup. J. Bangladesh Acad. Sci. 32(1): 55-60.

Majumdar, S., M. A. Hossain and K.K. Dey. 2011a. The endomorphism semigroup of an endomapping of a finite set. Ganit 31: 71-77.

Majumdar, S., K.K. Dey and M. A. Hossain. 2011b. Direct product and wreath product of transformation semigroups. Ganit 31: 1-7.

Meldrum, J. D. P. 1995. Wreath products of groups and semigroups. Longman.

Petrich, M. 1984. Inverse semigroups. John Wiley and Sons, New York. 